\title{
ON THE LOCATION OF THE ZEROS OF THE DERIVATIVE OF RATIONAL FUNCTIONS OF DISTANCE POLYNOMIALS
}

\author{
BY \\ AUGUSTA SCHURRER(')
}

1. Introduction. Nagy [6] $\left({ }^{2}\right)$ studied the class of polynomials in $E_{m}$ consisting of all expressions of the type (1.1).

$$
\begin{aligned}
& F\left(x_{1}, x_{2}, \cdots, x_{m}\right) \\
& \quad=c \prod_{k=1}^{n}\left[\left(x_{1}-x_{1, k}\right)^{2}+\left(x_{2}-x_{2, k}\right)^{2}+\cdots+\left(x_{m}-x_{m, k}\right)^{2}\right], \quad c>0 .
\end{aligned}
$$

The function $F$, to which the name of "distance polynomial" was given, is a non-negative, real function of the form $c\left(\sum_{i=1}^{n} x_{i}^{2 n}\right)+\Phi\left(x_{1}, x_{2}, \cdots, x_{m}\right)$ where $\Phi$ is a real polynomial of degree at most $2 n-1$. The "derivative" of $F$ was defined to be

$$
F^{\prime}\left(x_{1}, x_{2}, \cdots, x_{m}\right)=\sum_{h=1}^{n} F_{x_{h}}^{2} / 4 F, \quad F_{x_{h}}=\frac{\partial F}{\partial x_{h}},
$$

Nagy extended some theorems of Gauss, Lucas, Jensen, and Laguerre, concerning the location of the zeros of the derivative of a polynomial in two real variables, to the class of distance polynomials in $E_{m}$.

In this paper several other results concerning the geometry of the zeros of a polynomial in a single complex variable are extended to $E_{n}$. It is found convenient to introduce vector methods. $1 / A=A / A \cdot A=A / A^{2}$ denotes a particular reciprocal with respect to scalar multiplication; $\|A\|=[A \cdot A]^{1 / 2}$ the norm or length of the vector $A ; Q: w$ the point $Q$ with position vector $w$; $\mathbf{e}_{i} i=1,2, \cdots, n$ a basis for $E_{n}$.

(1.3) is a distance polynomial of degree $r$ in $E_{n}$.

$$
\begin{aligned}
F\left(x_{1}, x_{2}, \cdots, x_{n}\right) & =c \prod_{j=1}^{p}\left\|v-v_{j}\right\|^{2 m_{j}}=c \prod_{j=1}^{p} d_{j}^{m_{j}}\left(x_{1}, x_{2}, \cdots, x_{n}\right), c>0, \\
\sum_{j=1}^{p} m_{j} & =r . \quad v=\sum_{i=1}^{n} x_{i} e_{i} . \quad v_{j}=\sum_{i=1}^{n} x_{i}{ }^{(j)} e_{i} . \\
d_{j} & =\left\|v-v_{j}\right\|^{2} .
\end{aligned}
$$

Presented to the Society September 4, 1952; received by the editors January 18, 1957.

(1) These results were obtained in a doctoral dissertation completed under the direction of Professor Morris Marden of the University of Wisconsin.

(2) [6] indicates item 6 in the attached bibliography. 
Its derivative is (1.4).

$$
F^{\prime}\left(x_{1}, x_{2}, \cdots, x_{n}\right)=\frac{\|\nabla F\|^{2}}{4 F}=\frac{F}{4}\|\nabla \log F\|^{2}
$$

where $\nabla F=\sum_{i=1}^{n} F_{x_{i}} e_{i}$ and $F_{x_{i}}=\partial F / \partial x_{i}$.

$$
\begin{array}{r}
R\left(x_{1}, x_{2}, \cdots x_{n}\right)=\prod_{j=0}^{q} F_{j}\left(x_{1}, x_{2}, \cdots, x_{n}\right) / \prod_{j=q+1}^{p} F_{j}\left(x_{1} x_{2}, \cdots, x_{n}\right) \\
0 \leqq q \leqq p
\end{array}
$$

and $F_{0}\left(x_{1}, x_{2}, \cdots, x_{n}\right)=1$.

(1.5) is a rational function of the distance polynomials $F_{j}$. It will be assumed that $R$ has been "reduced to lowest terms," i.e. that $\prod_{j=0}^{q} F_{j}$ and $\prod_{j=q+1}^{p} F_{j}$ have no zeros in common. The "derivative" of (1.5) is

$$
R^{\prime}=\frac{\|\nabla R\|^{2}}{4 R}=\frac{R}{4}\|\nabla \log R\|^{2}
$$

where $\nabla R=\sum_{i=1}^{n} R_{x_{i}} e_{i}$ and $R_{x_{i}}=\partial R / \partial x_{i}$.

Theorem I, the central result of this paper, is an extension to the class of distance polynomials in $E_{n}$ of a theorem due to Marden [4, Theorem I].

Theorem I. Let $F_{j}\left(x_{1}, x_{2}, \cdots, x_{n}\right)$ be a distance polynomial of degree $n_{j}$ all of whose zeros lie in the spherical region $\sigma_{j} S_{j}(v) \equiv \sigma_{j}\left[\left(v-c_{j}\right)^{2}-r_{j}^{2}\right] \leqq 0, r_{j}>0$ and $\sigma_{j}= \pm 1$ for $j=1,2, \cdots, p$. Then every finite zero $P: V$ of $R^{\prime}(1.6)$ is such that $V$ satisfies at least one of the inequalities

$$
\sigma_{j} S_{j}(v) \leqq 0,
$$

$$
j=1,2, \cdots, p
$$

or

$$
E(v) / \prod_{j=1}^{p} S_{j}(v)=\sum_{j=1}^{p} \frac{N N_{j}}{S_{j}(v)}-\sum_{j=1, k=j+1}^{p} \frac{N_{j} N_{k} T_{j k}}{S_{j}(v) S_{k}(v)} \leqq 0
$$

where $N_{j}=\nu_{j} n_{j}, \nu_{j}=1$ for $j \leqq q$ and $\nu_{j}=-1$ for $j>q$, i.e., $N_{j}$ and $n_{j}$ are the signed and unsigned degrees respectively of $F_{j} . N=\sum_{k=1}^{p} N_{j}$ is the total degree of $R$. $\tau_{j k}=\left\|c_{j}-c_{k}\right\|^{2}-\left(\lambda_{j} r_{j}-\lambda_{k} r_{k}\right)^{2}$ where $\lambda_{j}=\nu_{j} \sigma_{j}$.

This theorem determines a closed region of space, bounded by the surface $E(v)=0$, which contains all the zeros of $R^{\prime} / R$, the "logarithmic derivative" of (1.5), as soon as the spherical regions over which the zeros of the $F_{j}$ are distributed are known. The class of spherical regions consists of the closed interiors and exteriors of spheres as well as of closed half spaces. This Mardentype theorem, applied to finite products and quotients, yields sharper results than those arising from the application of the extended Gauss-Lucas type theorem of Nagy [6, Theorem I]. 
2. Some lemmas. In the subsequent work one finds the following lemmas useful.

Lemma I. Let (1) $P: V, Q_{1}: v_{1}$, and $Q_{1}^{\prime}: w_{1}=1 /\left(V-v_{1}\right) \neq 0$ be points in $E_{n}$.

(2) $S(v) \equiv(v-c)^{2}-r^{2}=0, r>0$, and $S^{\prime}(v) \equiv\left(v-c^{\prime}\right)^{2}-r^{\prime 2}=0, r^{\prime}>0$, be the equations of two spheres in $E_{n}$ with center and radius

$$
C: \mathrm{c}, r \quad \text { and } \quad C^{\prime}: \mathrm{c}^{\prime}=V-\mathrm{c} / S(V), \quad r^{\prime}=r /|S(V)|
$$

respectively. Then the point $Q_{1}^{\prime}$ lies (1) inside or outside the sphere $S^{\prime}$ according as the sphere $S$ does or does not separate the two points $P$ and $Q_{1}$, or

(2) on the sphere $S^{\prime}$ if $S$ passes through $Q_{1}$ and not through $P$, and conversely.

Proof.

$$
\begin{aligned}
S^{\prime}\left(w_{1}\right) & =\left(w_{1}-\mathrm{c}^{\prime}\right)^{2}-r^{\prime 2} \\
& =\left[\frac{1}{V-v_{1}}-\frac{V-\mathrm{c}}{S(V)}\right]^{2}-\frac{r^{2}}{|S(V)|^{2}} \\
& =\frac{1}{\left(V-v_{1}\right)^{2}}-2\left[\frac{V-\mathrm{c}}{S(V)}\right] \cdot\left[\frac{V-v_{1}}{\left(V-v_{1}\right)^{2}}\right]+\frac{S(V)}{S^{2}(V)} \\
& =\frac{S(V)-2(V-\mathrm{c}) \cdot\left(V-v_{1}\right)}{S(V)\left(V-v_{1}\right)^{2}}+\frac{1}{S(V)} \\
& =\frac{S\left(v_{1}\right)}{S(V)} \mathbf{w}_{1}^{2} .
\end{aligned}
$$

$w_{1}^{2} \geqq 0 . w_{1}^{2}=0$ if, and only if, $w_{1}=0$. Since $w_{1} \neq 0$, the sign of $S^{\prime}\left(w_{1}\right)$ is the same as that of $S\left(v_{1}\right) / S(V)$. It follows that if

(1) $S(v)=0$ separates $P$ and $Q_{1}, S(V)$ and $S\left(v_{1}\right)$ are opposite in sign and $S^{\prime}\left(w_{1}\right)<0 . Q_{1}^{\prime}$ is in the sphere $S^{\prime}(v)=0$.

(2) the points $P$ and $Q_{1}$ are both exterior to or interior to $S(v)=0, S(V)$ and $S\left(v_{1}\right)$ are similarly signed and $S^{\prime}\left(w_{1}\right)>0 . Q_{1}^{\prime}$ is outside of the sphere $S^{\prime}(v)=0$.

(3) $S$ passes through $Q_{1}, S\left(v_{1}\right)=0$. If $S$ does not pass through $P, S(V) \neq 0$. If $P$ is understood to be distinct from $Q_{1}$, it follows that $S^{\prime}\left(w_{1}\right)=0$, i.e. $S^{\prime}$ passes through $Q_{1}^{\prime}$.

Starting with any one of the statements $S^{\prime}\left(w_{1}\right)<0, S^{\prime}\left(w_{1}\right)>0$, or $S^{\prime}\left(w_{1}\right)$ $=0$, one may without difficulty retrace his steps and establish the converse.

Lemma II. If for $j=1,2, \cdots, p$ the points $Q_{j}: w_{j}$ vary independently over the closed interiors of the spheres $S_{j}(v) \equiv\left(v-c_{j}\right)^{2}-r_{j}^{2}=0, r_{j}>0$, the locus of the point $Q: w=\sum_{j=1}^{p} m_{j} w_{j}$ where the $m_{j}$ are real will be the closed interior of the sphere $S(v)=0$ of radius $r=\sum_{j=1}^{p}\left|m_{j}\right| r_{j}$ and center $C: c=\sum_{j=1}^{p} m_{j} \mathbf{c}_{j}$. 
Proof.

$$
\|\boldsymbol{w}-\mathrm{c}\|=\left\|\sum_{j=1}^{p} m_{j}\left(\boldsymbol{w}_{j}-\mathrm{c}_{j}\right)\right\| \leqq \sum_{j=1}^{p}\left|m_{j}\right|\left\|\boldsymbol{w}_{j}-\mathrm{c}_{j}\right\| .
$$

By hypothesis $Q_{j}$ is in the closed interior of $S_{j}$, i.e., $\left\|w_{j}-c_{j}\right\| \leqq r_{j}$, for $j$ $=1,2, \cdots, p$. It follows that $\|w-c\| \leqq \sum_{j=1}^{p}\left|m_{j}\right| r_{j}=r$ and $(w-c)^{2}-r^{2}$ $\leqq 0$. Consequently $Q$ lies in the closed interior of the sphere $S(v)=0$.

Conversely, it can be established that if $Q: w$ lies in the closed interior of $S(v)=0$, there exists a point $Q_{j}: w_{j}$ in each of the spheres $S_{j}(v)=0, j$ $=1,2, \cdots, p$, such that $w=\sum_{j=1}^{p} m_{j} w_{j}$.

If $Q: w=\sum_{i=1}^{n} w_{i} e_{i}$ lies in the closed interior of $S(v)=0$ and $c=\sum_{i=1}^{n} \alpha_{i} \boldsymbol{e}_{i}$, $w-c=\sigma r \sum_{i=1}^{n} \lambda_{i} e_{i}$ where $0 \leqq \sigma \leqq 1$ and $\lambda_{i}=\left(w_{i}-\alpha_{i}\right)\left[\sum_{i=1}^{n}\left(w_{i}-\alpha_{i}\right)^{2}\right]^{-1 / 2}$.

Consider the set of points $Q_{j}: w_{j}=c_{j}+\left(\sigma\left|m_{j}\right| / m_{j}\right) r_{j} \sum_{i=1}^{n} \lambda_{i} e_{i}, 0 \leqq \sigma \leqq 1$, $j=1,2, \cdots, p$. Since $\sum_{i=1}^{n} \lambda_{i}^{2}=1$ and $e_{i} \cdot e_{j}=\delta_{i j},\left(w_{j}-c_{j}\right)^{2}=\sigma^{2} r_{j}^{2}\left(\sum_{i=1}^{n} \lambda_{i}^{2}\right)$ $\leqq r_{j}^{2}$. Consequently $Q_{j}: w_{j}$ lies in the closed interior of the sphere $S_{j}(v)=0$. Moreover, since

$$
\begin{aligned}
\sum_{j=1}^{p} m_{j} \boldsymbol{w}_{j} & =\sum_{j=1}^{p} m_{j} \mathrm{c}_{j}+\sigma\left(\sum_{j=1}^{p}\left|m_{j}\right| r_{j} \mid\right)\left(\sum_{i=1}^{n} \lambda_{i} \boldsymbol{e}_{i}\right)=\mathrm{c}+\sigma r\left(\sum_{i=1}^{n} \lambda_{i} \boldsymbol{e}_{i}\right) \\
& =\mathrm{c}+(\boldsymbol{w}-\mathrm{c})=\mathrm{w},
\end{aligned}
$$

the desired locus is the closed interior of the sphere $S(v)=0$.

Lemma II is the $n$ space analogue of Lemma $\mathrm{I}$ in Marden [4].

Lemma III. If $P: V$ is a finite zero of $R^{\prime} / R$, the "logarithmic derivative" of the rational function (1.5) of the distance polynomials $F_{j}, F_{j}\left(x_{1}, x_{2}, \cdots, x_{n}\right)$ $=c_{j} \prod_{\delta_{j-1}+1}^{\delta_{j}} d_{k}^{m_{k}}\left(x_{1}, x_{2}, \cdots, x_{n}\right), \delta_{0}=0, c_{j}>0, d_{k}=\left\|v-v_{k}\right\|^{2}, m_{k}>0$ and real, $j=1,2, \cdots, p, V$ must satisfy $(2.1)$.

$$
\sum_{k=1}^{\delta_{p}} \frac{M_{k}}{V-v_{k}}=0,
$$

$M_{k}=\mu_{k} m_{k} . \mu_{k}=1$ for $1 \leqq k \leqq q . \mu_{k}=-1$ for $q+1 \leqq k \leqq p$.

Proof. A necessary and sufficient condition that $P: V$ be a finite zero of $R^{\prime} / R$ is that $\nabla \log R$ vanish at $P . \nabla \log R=\sum_{j=1}^{q} \nabla \log F_{j}-\sum_{j=q+1}^{p} \nabla \log F_{j}$ $=\sum_{j=1}^{p} \mu_{j} \nabla \log F_{j}$ where $\mu_{j}=1$ for $0 \leqq j \leqq q$ and $\mu_{j}=-1$ for $q+1 \leqq j \leqq p$. If $v=\sum_{i=1}^{n} x_{i} e_{i}$ and $v_{k}=\sum_{i=1}^{n} x_{i}^{(k)} e_{i}, d_{k}=\left\|v-v_{k}\right\|^{2}=\sum_{i=1}^{n}\left(x_{i}-x_{i}^{(k)}\right)^{2}$.

$$
\log F_{j}=\log c_{j}+\sum_{k=\delta_{j-1}+1}^{\delta j} m_{k} \log d_{k}
$$

and therefore

$$
\frac{\partial \log F_{j}}{\partial x_{i}}=\sum_{k=\delta_{j-1}+1}^{\delta_{j}} m_{k}\left(\frac{\partial \log d_{k}}{\partial x_{i}}\right)=\sum_{k=\delta_{j-1}+1}^{\delta_{j}} \frac{2 m\left(x_{i}-x_{i}^{(k)}\right)}{d_{k}} .
$$


Since

$$
\begin{aligned}
\nabla \log F_{j} & =\sum_{i=1}^{n} \frac{\partial \log F_{j}}{\partial x_{i}} \boldsymbol{e}_{i}=\sum_{k=\delta_{j-1}+1}^{\delta_{j}} \frac{2 m_{k}\left[\sum_{i=1}^{n}\left(x_{i}-x_{i}^{(k)}\right) \boldsymbol{e}_{i}\right]}{d_{k}} \\
& =\sum_{k=\delta_{j-1}+1}^{\delta_{j}} \frac{2 m_{k}}{v-v_{k}}, \quad \nabla \log R=\sum_{j=1}^{p} \mu_{j}\left(\sum_{k=\delta_{j-1}+1}^{\delta_{j}} \frac{2 m_{k}}{v-v_{k}}\right)
\end{aligned}
$$

and (2.2) holds.

$$
\nabla \log R=\sum_{k=1}^{\delta_{p}} \frac{2 M_{k}}{v-v_{k}}, \quad \quad M_{k}=\mu_{k} m_{k} .
$$

Clearly $V$ must satisfy (2.1).

LEMMA IV. If (1) $Q_{j}: V_{j}$ lies in the spherical region $\sigma_{j} S_{j}(v) \equiv \sigma_{j}\left[\left(v-c_{j}\right)^{2}-r_{j}^{2}\right]$ $\leqq 0$,

$$
r_{j}>0, \sigma= \pm 1, \quad j=1,2, \cdots, p .
$$
and

(2) $P: V$ is exterior to all of the spherical regions $\sigma_{j} S_{j}(v) \leqq 0, j=1,2, \cdots, p$

(3) $\sum_{j=1}^{p} m_{j} /\left(V-v_{j}\right)=0, m_{j}$ real, $V$ must satisfy inequality (2.3).

$$
I(v) \equiv\left[\sum_{j=1}^{p} \frac{m_{j}\left(v-c_{j}\right)}{S_{j}(v)}\right]^{2}-\left[\sum_{j=1}^{p} \frac{\left|m_{j}\right| r_{j}}{\left|S_{j}(v)\right|}\right]^{2} \leqq 0 .
$$

Proof. By hypothesis $S_{j}(v)=0$ separates $Q_{j}$ and $P$. It follows from Lemma I that the point $Q_{j}^{\prime}: w_{j}=1\left(V-v_{j}\right)$ is in the sphere

$$
S_{j}^{\prime}(v) \equiv\left[v-\frac{\left(V-c_{j}\right)}{S_{j}(V)}\right]^{2}-\left[\frac{r_{j}}{\left|S_{j}(V)\right|}\right]^{2}=0 .
$$

If we let $w=\sum_{j=1}^{n} m_{j} w_{j}$ where the $m_{j}$ are real, by Lemma II, the locus of $Q: w$ is the closed interior of the sphere $T(v)=0$, i.e. (2.4) holds.

$$
T(w) \equiv\left(w-\sum_{j=1}^{p} \frac{m_{j}\left(V-c_{j}\right)}{S_{j}(V)}\right)^{2}-\left(\sum_{j=1}^{p} \frac{\left|m_{j}\right| r_{j}}{\left|S_{j}(V)\right|}\right)^{2} \leqq 0 .
$$

By hypothesis $0=\sum_{j=1}^{p} m_{j} /\left(V-v_{j}\right)=\sum_{j=1}^{p} m_{j} w_{j}=w$. Therefore $w=0$ is a value corresponding to a set of suitably chosen points $Q_{j}$ in the given regions $\sigma_{j} S_{j}(v) \leqq 0 . w=0$ must satisfy (2.4) and it follows that, under the hypotheses of this lemma, (2.3) holds.

3. A Marden-type theorem. Theorem I can now be established.

Proof. Let $P: V$ be a finite zero of $R^{\prime}$. If $P$ is also a zero of $R, P$ must coincide with at least one zero of some $F_{j}$ for $1 \leqq j \leqq q$. Therefore it must lie in at 
least one of the given spherical regions and (1.7) holds. If $P$ is not a zero of $R$, either $P$ lies in at least one of the given spherical regions, in which event $V$ satisfies at least one of the inequalities (1.7), or $P$ is exterior to all of the given regions. It will be shown that in the latter case $V$ satisfies (1.8).

Since $P$ is a zero of $R^{\prime}$ and not of $R$, it follows from Lemma III that $\sum_{k=1}^{\delta_{p}} M_{k} /\left(V-v_{k}\right)=0$ where $M_{k}=\mu_{k} m_{k}, M_{k} \neq 0$ and real, $m_{k}>0$ and real, $\mu_{k}=1$ for $k \leqq q$ and $\mu_{k}=-1$ for $k>q$.

It is convenient to first consider distance polynomials $F_{j}$ each of which has a single zero of multiplicity $n_{j}$ which lies in the spherical region $\sigma_{j} S_{j}(v) \leqq 0$ for $j=1,2, \cdots, p$, i.e.

$$
F_{j}\left(x_{1}, x_{2}, \cdots, x_{n}\right)=c_{j} d_{j}^{n_{j}}\left(x_{1}, x_{2}, \cdots, x_{n}\right),
$$

Since $P$ is exterior to all $p$ of the given spherical regions, Lemma IV applies and it follows that $V$ must satisfy inequality (2.3), $I(v) \leqq 0$, with $N_{j}$ $=\nu_{j} n_{j}$ replacing $m_{j} . \nu_{j}=1$ for $j \leqq q$ and $\nu_{j}=-1$ for $j>q$. When $I(v)$ is expanded and simplified it becomes

$$
I(v) \equiv \sum_{j=1}^{p} \frac{N_{j}^{2}}{S_{j}(v)}+\sum_{j=1, k=j+1}^{p} \frac{2 N_{j} N_{k}\left[\left(v-c_{j}\right) \cdot\left(v-c_{k}\right)-\lambda_{j} \lambda_{k} r_{j} r_{k}\right]}{S_{j}(v) S_{k}(v)}
$$

where $\lambda_{j}=\mu_{j} \sigma_{j}$.

Since $\left(c_{j}-c_{k}\right)^{2}=\left(v-c_{j}\right)^{2}+\left(v-c_{k}\right)^{2}-2\left(v-c_{j}\right) \cdot\left(v-c_{k}\right)$ and $\left(\lambda_{j} r_{j}-\lambda_{k} r_{k}\right)^{2}$ $=r_{j}^{2}+r_{k}^{2}-2 \lambda_{j} \lambda_{k} r_{j} r_{k}, 2\left[\left(v-c_{j}\right) \cdot\left(v-c_{k}\right)-\lambda_{j} \lambda_{k} r_{j} r_{k}\right]=S_{j}(v)+S_{k}(v)-\tau_{j k}$ where $\tau_{j k}$ $=\left[\left(\mathrm{c}_{j}-\mathrm{c}_{k}\right)^{2}-\left(\lambda_{j} r_{j}-\lambda_{k} r_{k}\right)^{2}\right]$.

If $\sum_{j=1}^{p} N_{j}$ is replaced by $N,(3.2)$ reduces to (3.3)

$$
I(v)=\sum_{j=1}^{p} \frac{N N_{j}}{S_{j}(v)}-\sum_{j=1, k=j+1}^{p} \frac{N_{j} N_{k} \tau_{j k}}{S_{j}(v) S_{k}(v)} .
$$

It follows that $V$ must satisfy condition (1.8).

Now consider the distance polynomials (3.4)

$$
\begin{aligned}
F_{j}\left(x_{1}, x_{2}, \cdots, x_{n}\right) \\
=c_{j} \prod_{k=1}^{p} d_{j k}^{m_{j k}}\left(x_{1}, x_{2}, \cdots, x_{n}\right), j=1,2, \cdots, p, \quad c_{j}>0
\end{aligned}
$$

each of which has all of its zeros in the spherical region $\sigma_{j} S_{j}^{*}(v) \leqq 0$ where $S_{i}^{*}(v) \equiv\left(v-c_{j}^{*}\right)^{2}-r_{j}^{* 2}, r_{j}^{*}>0$. Let $Q_{m k}$ be the zero corresponding to the first degree distance polynomial $d_{m k}$. The zeros $Q_{m k}, k=1,2, \cdots, p_{m}$, of $F_{m}$ all lie in $\sigma_{m} S_{m}^{*}(v) \leqq 0$. This is a specialization of the preceding case in which the $p_{m}$ spheres $S_{j}$ coalesce in the sphere $S_{m}^{*}$. The derivation of the desired vector inequalities follows as above.

The boundary surface $E(v)=0$ of the region of space described by the inequality (1.8) can be written in the form $\left(\sum_{t=1}^{n} x_{i}^{2}\right)^{p}+\Phi\left(x_{1}, x_{2}, \cdots, x_{n}\right)=0$ where $\Phi$ is a real polynomial of degree at most $2 p-1$. In $E_{3}$ this surface con- 
tains the circle at infinity as a $p$-fold curve and it will be referred to as a $p$ spherical $2 p-i c$ surface. When $p=2$ the surface is a cyclide. These surfaces were studied in detail by Darboux [2].

It is not difficult to show that in certain cases when the spheres $S_{j}$ are all symmetrically located with respect to a fixed point 0 and $\sigma_{j}=1$ for all $j$, the surface $E(v)=0$ degenerates into a set of spheres centered at 0 , and the desired region $E(v) \leqq 0$ is the closed interior of the largest sphere in that set.

4. The zeros of $R-\lambda^{2} R^{\prime}$. An interesting application of Theorem I is displayed in the next result.

THEOREM II. Under the hypotheses of Theorem I every finite zero of $R-\lambda^{2} R^{\prime}$, $\lambda$ real, satisfies at least one of the $p+1$ inequalities

$$
\begin{array}{rlrl}
\sigma_{j} S_{j}(v) & \leqq 0, & j & =1,2, \cdots, p . \\
\frac{M(v)}{\prod_{j=1}^{p} S_{j}(v)}=\sum_{j=1}^{p} \frac{N_{j} \lambda}{N S_{j}(v)} T_{j}(v)-\sum_{j=1, k=j+1}^{p} \frac{N_{j} N_{k} \tau_{j k}}{S_{j}(v) S_{k}(v)} & \leqq 0
\end{array}
$$

where $T_{j}(v)=\left[v-\left(c_{j}+N w\right)\right]^{2}-r_{j}^{2}, w=\lambda e$, and $\boldsymbol{e}$ is the unit vector in the $\nabla R$ direction.

Proof. Let $P: V$ be a zero of $R-\lambda^{2} R^{\prime}$. If $P$ is a zero of $R$, it is also a zero of $R^{\prime}$ and $V$ corresponds to a point which lies in at least one of the spherical regions described by (4.1). If $P$ is not a zero of $R$, either $P$ lies in one of the given spherical regions, in which case $V$ satisfies at least one of the vector inequalities (4.1), or $P$ is exterior to all of these regions. In the latter event it will be shown that $V$ must satisfy (4.2).

Since $P$ is a zero of $R-\lambda^{2} R^{\prime}$ and not of $R$,

$$
\left.\frac{R^{\prime}}{R}\right|_{P}=\left.\frac{\|\nabla R\|^{2}}{4 R^{2}}\right|_{P}=\frac{1}{4}\|\nabla \log R\|_{P}^{2}=\lambda^{-2}
$$

and from (2.2) one obtains

$$
\left.\frac{1}{2} \frac{\nabla R}{R}\right|_{P}=\left.\frac{\nabla \log R}{2}\right|_{P}=\sum_{j=1}^{p} \frac{N_{j}}{V-v_{j}}=\frac{1}{\lambda} \mathrm{e}
$$

where $\boldsymbol{e}$ is the unit vector in the $\nabla R$ direction.

Since $P$ is exterior to all of the given spherical regions one can proceed as in the proof of Lemma II and it follows that $\sum_{j=1}^{p} N_{j} / V-v_{j}=w$, a value corresponding to a suitably chosen set of points $Q_{j}: w_{j} j=1,2, \cdots, p$, each of which lies in the corresponding spherical region $\sigma_{j} S_{j}(v) \leqq 0$, must satisfy (2.4). That is

$$
\left(w-\sum_{j=1}^{p} N_{j}\left(V-c_{j}\right) / S_{j}(V)\right)^{2}-\left(\sum_{j=1}^{p}\left|N_{j}\right| \sigma_{j} r_{j} / S_{j}(V)\right)^{2} \leqq 0 .
$$


When the left hand side is expanded this inequality reduces to (4.2). $T_{j}(v)=0$ is the sphere obtained by translating $S_{j}(v)=0$ in the direction of $N \lambda e$ by an amount equal to the magnitude of that vector.

Corollary. If all the zeros of a distance polynomial $F\left(x_{1}, x_{2}, \cdots, x_{n}\right)$ of degree $m$ lie in a sphere $S_{1}(v)=0$, any zero of $F-\lambda^{2} F^{\prime}, \lambda$ real, will lie either in $S_{1}$ or in the sphere obtained by translating $S_{1}$ in the direction of $m \lambda e$ by an amount equal to the magnitude of that vector. $e$ is the unit vector in the $\nabla F$ direction.

Proof. If $p=q=1, \sigma_{1}=1$, i.e. $R=F$, a distance polynomial of degree $m$ with all of its zeros in $S_{1}(v)=0$, it follows from Theorem II that the zeros of $F-\lambda^{2} F^{\prime}$ either lie in $S_{1}$ or are such that their position vectors satisfy (4.2). In this case (4.2) reduces to $m w^{2} T(V) / m S_{1}(V) \leqq 0$. Since $P$ is exterior to $S_{1}, S_{1}(V)>0$ and consequently $T_{1}(V)=\left[V-\left\{c_{1}+m \lambda e\right\}\right]^{2}-r_{1}^{2} \leqq 0$. [4].

The results of this section are generalizations of Theorem II in Marden

5. The critical points of finite products of distance polynomials in $E_{3}$. Nagy [6] developed the following Gauss-Lucas type theorem for the class of distance polynomials in $E_{3}$ : "Let $F\left(x_{1}, x_{2}, x_{3}\right)$ be a distance polynomial with zeros $Q_{j}, j=1,2, \cdots, n$, and let $K$ be the smallest convex region of space which contains those zeros. Then all the zeros of $F^{\prime}$ also lie in $K$. No zero of $F^{\prime}$ is on the boundary of $K$ unless it is a multiple zero of $F$ or unless all the $Q_{j}$ are coplanar."

As soon as the location of all the zeros of $F$ is known Nagy's theorem singles out a portion of space which must contain the zeros of the logarithmic derivative of $F$. If $F$ is a finite product of distance polynomials, Theorem I will restrict even further the region of space within which the critical points may lie.

Let $F\left(x_{1}, x_{2}, x_{3}\right)=\prod_{j=1}^{p} F_{j}\left(x_{1}, x_{2}, x_{3}\right)$ be a finite product of the distance $F_{j}$ where each $F_{j}$ is of degree $n_{j}$ and has all of its zeros in the closed interior of the sphere $S_{j}$. In this section the region containing the critical points of $F$ is determined for $p=2$ and $p=3$ when the spheres in question have collinear centers and a common external center of similitude. In each case the desired region consists not only of the closed interiors of the given spheres but also of the closed interiors of another set of spheres having the same external center of similitude as the given set. The centers of the second set are located at the zeros of the logarithmic derivative of the distance polynomial $G\left(x_{1}, x_{2}, x_{3}\right)$ obtained from $F$ by coalescing all the zeros of each $F_{j}$ at the center of the corresponding sphere $S_{j}$.

THEOREM III. If $F\left(x_{1}, x_{2}, x_{3}\right)$ is an nth degree distance polynomial $n_{1}$ of whose zeros lie in the closed interior of the sphere $S_{1}$ and the remainder of whose zeros, $n_{2}=n-n_{1}$ in number, lie in the closed interior of the sphere $S_{2}$, all the zeros of $F^{\prime}\left(x_{1}, x_{2}, x_{3}\right)$ lie either in the closed interior of $S_{1}$ or $S_{2}$, or in the closed interior of a third sphere $S(v)=0$ where 


$$
S(v) \equiv\left(v-\frac{n_{1} c_{2}+n_{2} c_{1}}{n_{1}+n_{2}}\right)^{2}-\left(\frac{n_{1} r_{2}+n_{2} r_{1}}{n_{1}+n_{2}}\right)^{2}
$$

$S$ has a common center of similitude with $S_{1}$ and $S_{2}$ and its center is located at the centroid of a system of two particles, one of mass $n_{2}$ located at the center of the sphere $S_{1}$ and the other of mass $n_{1}$ located at the center of the sphere $S_{2}$.

Proof. This is a special case of Theorem I for which $q=2 ; N_{j}=n_{j} j=1,2$; $N=n_{1}+n_{2} ; \nu_{i}=1, \sigma_{i}=1, \lambda_{i}=1$ for $i=1,2$.

$F\left(x_{1}, x_{2}, x_{3}\right)=F_{1}\left(x_{1}, x_{2}, x_{3}\right) F_{2}\left(x_{1}, x_{2}, x_{3}\right)$. Each $F_{j}$, a distance polynomial of degree $n_{j}$, has all of its zeros in the closed interior of the sphere $S_{j}$. As a consequence of Theorem I $P: V$, a zero of $F^{\prime}$, lies either in the closed interior of $S_{1}$ or $S_{2}$ or, if $P$ is exterior to both of these spheres, $V$ satisfies (1.8) which in this case reduces to

$$
\left(n_{1}+n_{2}\right)\left[\frac{n_{1}}{S_{1}(V)}+\frac{n_{2}}{S_{2}(V)}\right]-\frac{n_{1} n_{2} \tau_{12}}{S_{1}(V) S_{2}(V)} \leqq 0 .
$$

If both sides of the inequality are multiplied by $S_{1}(V) S_{2}(V)>0$ and the $S_{j}$ and $\tau_{12}$ are replaced by their equivalents, this condition reduces to

$$
\left[\left(n_{1}+n_{2}\right) \boldsymbol{V}-\left(n_{1} c_{2}+n_{2} c_{1}\right)\right]^{2}-\left(n_{1} r_{2}+n_{2} r_{1}\right)^{2}=\left(n_{1}+n_{2}\right)^{2} S(V) \leqq 0 .
$$

Consequently if $P$ is exterior to both $S_{1}$ and $S_{2}$, it must lie in the closed interior of $S$.

This theorem is an extension of a result due to Walsh [7].

TheOREM IV. If all the zeros of the distance polynomial $F_{j}\left(x_{1}, x_{2}, x_{3}\right)$ $=\prod_{k=1}^{p_{j}}\left\|_{v}-v_{k}\right\| 2 m_{k}$ of degree $n_{j}, j=1,2,3$, lie in the closed interior of the sphere $S_{j}$ and if all the $S_{j}$ have a common external center of similitude 0 , then each zero of the derivative of $F=\prod_{j=1}^{3} F_{j}\left(x_{1}, x_{2}, x_{3}\right)$ lies either in the closed interior of one of the $S_{j}$ or in the closed interior of one of the spheres $S_{1}^{\prime}$ or $S_{2}^{\prime}$. The spheres $S_{1}^{\prime}$ and $S_{2}^{\prime}$ also have the external center of similitude 0 and their centers correspond to the zeros of the logarithmic derivative of the distance polynomial $G(v)$ $=\prod_{j=1}^{3} G_{j}(v)=\prod_{j=1}^{3}\left\|_{v}-c_{j}\right\|^{2 n_{i}}$ where the $c_{j}$ are the position vectors of the centers $C_{j}$ of the spheres $S_{j}$.

Proof. Without any loss of generality the line of centers may be taken as the $x_{1}$ axis and the center of similitiude 0 as the origin of a rectangular coordinate system. $C_{j}: c_{j}$ where $c_{j}=c_{j} \boldsymbol{\theta}_{1}$ is the center of $S_{j}$. Since 0 is a center of similitude $r_{j} c_{j}^{-1}=\lambda$ for $j=1,2,3$. It follows that $S_{j}$ has the form $S_{j} \equiv\left(\sum_{i=1}^{3} x_{i}^{2}\right)$ $-2 c_{j} x_{1}+\mu x_{1}^{2}$ and $\tau_{j k}=\left\|c_{j}-c_{k}\right\|^{2}-\left(r_{j}-r_{k}\right)^{2}=\mu\left(c_{j}-c_{k}\right)^{2}$ where $\mu=1-\lambda^{2}$.

As a consequence of Theorem I all the zeros $P: V$ of $F^{\prime}$ lie either in one of the given spheres $S_{j}$ or in the closed region of space described by (1.8). Under these hypotheses $p=3, \nu_{j}=1, \sigma_{j}=1, \lambda_{j}=1$, and consequently $N_{j}=n_{j}$ for $j=1,2,3$. If $P$ is exterior to all of the $S_{j}, \Pi_{j=1}^{3} S_{j}(V)>0$ and (1.8) reduces to 


$$
\sum_{i, j, k,-1 ; i \neq j \neq k}^{3}\left[N N_{i} S_{j}(V) S_{k}(V)-\mu N_{j} N_{k}\left(\mathbf{c}_{j}-\mathrm{c}_{k}\right)^{2} S_{i}(V)\right] \leqq 0
$$

where $N=\sum_{i=1}^{3} N_{i}$. The boundary surface of the desired region of space is clearly of order 4 .

After substituting the appropriate expressions for the $S_{j}$, the equation of the boundary surface, $E(v)=0$, reduces to

$$
n^{2}\left(\sum_{i=1}^{3} x_{i}^{2}\right)^{2}-\left(2 A x_{1}-B\right)\left(\sum_{i=1}^{3} x_{i}^{2}\right)+4 C x_{1}^{2}-2 D x_{1}+E=0
$$

where

$$
\begin{aligned}
& A=n \sum_{i=1}^{3}\left(n-n_{i}\right) c_{i} ; \\
& B=\mu\left[\left\{\sum_{i=1}^{3}\left(n-n_{i}\right)^{2} c_{i}^{2}\right\}+2\left(n_{1} c_{1}+n_{2} c_{2}\right) n_{3} c_{3}\right] \\
& C=n\left\{\sum_{i, j, k=1 ; i \neq j \neq k ; j<k}^{3} n_{i} c_{j} c_{k}\right\} ; \\
& D=\mu\left[\sum_{i, j, k=1 ; i \neq j \neq k, j<k}^{3} n_{i} c_{j} c_{k}\left\{\left(n-n_{j}\right) c_{j}+\left(n-n_{k}\right) c_{k}\right\}+2 c_{1} c_{2} c_{3} \sum_{i, j=1 ; i \neq j, i<j}^{8} n_{i} n_{j}\right] ;
\end{aligned}
$$

and

$$
E=\mu^{2}\left[c_{1}^{2}\left(\sum_{i, j=1 ; i \neq j, i<j}^{3} n_{i}^{2} c_{j}^{2}\right)+2 c_{1} c_{2} c_{3}\left(\sum_{i, j, k=1 ; i \neq j \neq k ; j<k}^{3} c_{i} n_{j} n_{k}\right)\right] .
$$

Consider the function $G(v)=\prod_{j=1}^{3}\left\|v-c_{j}\right\|^{2 n_{i}}$. This function can be obtained by coalescing all the zeros of each $F_{j}$ at the center of the sphere $S_{j}$. The zeros of the logarithmic derivative of $G$ must satisfy the relation $\sum_{j=1}^{3}\left(n_{j} /\left(v-c_{j}\right)\right)=0$. Nagy's Lucas-type theorem, cited at the beginning of this section, shows that the zeros of $G^{\prime}(v)$ must lie on the $x_{1}$ axis. If $V$ corresponds to a zero of $G^{\prime}, V=X e_{1}$. The condition which must be satisfied by the zeros of the logarithmic derivative of $G$ may now be rewritten as $\sum_{j=1}^{3}\left(n_{j} /\left(X-c_{j}\right) e_{1}\right)=0$ which is equivalent to (5.2).

$$
n X^{2}-\left[\sum_{i=1}^{8}\left(n-n_{i}\right) c_{i}\right] X+\sum_{i, j, k=1 ; i \neq j \neq k, j<k}^{8} n_{i} c_{j} c_{k}=0 .
$$

If $\gamma_{1}$ and $\gamma_{2}$ are the zeros of (5.2), $n\left(\gamma_{1}+\gamma_{2}\right)=\sum_{i=1}^{3}\left(n-n_{i}\right) c_{i}$ and $n \gamma_{1} \gamma_{2}$ $=\left(\sum_{j, j, k-1 ; i \neq j \neq k, j<k}^{33} n_{i} c_{j} c_{k}\right)$. The spheres $S_{j}^{\prime}$ with centers $C_{j}^{\prime}: \gamma_{j} \Theta_{1}$ and with 0 as their external center of similitude are described by the equation

$$
S_{j}^{\prime}\left(x_{1}, x_{2}, x_{3}\right) \equiv\left(\sum_{i=1}^{3} x_{i}^{2}\right)-2 \gamma_{j} x_{1}+\mu \gamma_{j}^{2}=0, \quad j=1,2 .
$$


After forming the expression $n^{2} S_{1}^{\prime} S_{2}^{\prime}$ it is not difficult to show that it is identical to (5.1). In short, $E(v)=0$, the boundary surface in question, degenerates into two spheres $S_{1}^{\prime}$ and $S_{2}^{\prime}$ with centers whose position vectors correspond to the zeros of the logarithmic derivative of $G(v)$. The region of space described by $E(v) \equiv n^{2} S_{1}^{\prime}(v) S_{2}^{\prime}(v) \leqq 0$ consists of the closed interiors of the spheres $S_{1}^{\prime}$ and $S_{2}^{\prime}$ and contains all the zeros of $F^{\prime}$ which do not lie in any of the $S_{j}$.

By introducing a coordinate system with its origin at the center of $S_{1}$ and proceeding as in Theorem IV, the following result may be obtained.

Theorem V. Let $F\left(x_{1}, x_{2}, x_{3}\right)=\prod_{j=1}^{3} F_{j}\left(x_{1}, x_{2}, x_{3}\right)$ where each $F_{j}$ is a distance polynomial of degree $n_{j}$ all of whose zeros lie in the sphere $S_{j}$ of radius $r$ and center $C_{j}: c_{j}$. Then the zeros of $F^{\prime}$ lie either in the closed interiors of the given spheres $S_{j}$ or in the closed interiors of $S_{1}^{\prime}$ and $S_{2}^{\prime} . S_{1}^{\prime}$ and $S_{2}^{\prime}$ are spheres of radius $r$ whose centers correspond to the zeros of the logarithmic derivative of $G(v)=\prod_{j=1}^{3}\left\|v-c_{j}\right\|^{2 n_{i}}$.

The general case of both of these theorems was developed for polynomials in a single complex variable by Walsh $[8 \mathrm{~b}]$.

6. The critical points of finite quotients of distance polynomials in $E_{3}$. Proceeding as in Theorem III the following two theorems may easily be verified.

TheOREM VI. If the distance polynomial $F_{j}\left(x_{1}, x_{2}, x_{3}\right)$ of degree $n_{j}$ has all of its zeros in or on the sphere $S_{j}(v) \equiv\left(v-c_{j}\right)^{2}-r_{j}^{2}=0, r_{j}>0$, for $j=1,2, n_{1} \neq n_{2}$, all of the finite zeros of the derivative of the quotient $F=F_{1} / F_{2}$ lie in the closed interiors of $S_{1}, S_{2}$, and a third sphere

$$
S(v) \equiv\left(v-\frac{n_{2} c_{1}-n_{1} c_{2}}{n_{2}-n_{1}}\right)^{2}-\left(\frac{n_{2} r_{1}+n_{1} r_{2}}{\left|n_{2}-n_{1}\right|}\right)^{2}=0 .
$$

This is an extension of a result obtained by Walsh [8a].

Theorem VII. If the distance polynomial $F_{j}\left(x_{1}, x_{2}, x_{3}\right)$ of degree $n$ has all of its zeros in the spherical region $\sigma_{j} S_{j}(v) \leqq 0$ where $\sigma_{j}= \pm 1, j=1,2$, and the two spherical regions have no points in common, all the finite zeros of $F=F_{1} / F_{2}$ lie in the two given spherical regions.

This result was obtained by Bôcher [1] for a polynomial in a single complex variable.

The special case of Theorem I for which $N_{1}=n_{1}, N_{2}=n_{2}, N_{3}=-n_{3}$, and $N=\sum_{i=1}^{3} N_{i}=n_{1}+n_{2}-n_{3}=0$ leads to some interesting results which are summarized in the final two theorems.

THEOREM VIII. If the points $Q_{j}: \psi_{j}$ vary independently over the spherical regions $\sigma_{j} S_{j}(v) \leqq 0$ for $j=1,2,3$, any point $P: V$ whose position vector forms $a$ constant cross ratio with the $\psi_{j}$ lies in a fourth spherical region. 
Theorem IX. If (1) $F_{j}\left(x_{1}, x_{2}, x_{3}\right)$ is a distance polynomial of degree $n_{j}$ all of whose zeros lie in the spherical region $\sigma_{j} S_{j}(v) \leqq 0$ for $j=1,2,3,(2) n_{3}=n_{1}+n_{2}$ and (3) the given spherical regions have no point in common, every finite zero of the derivative of $F=F_{1} F_{2} / F_{3}$ is such that it lies in at least one of the given spherical regions or in a fourth spherical region. This fourth region is described by a point $P$ whose position vector $V$ forms a constant cross ratio, $-n_{2} / n_{1}$, with the position vectors $\psi_{j}$ of the points $Q_{j}$ which describe the given spherical regions $\sigma_{j} S_{j}(v) \leqq 0, j=1,2,3$.

Proof, Any zero $P: V$ of $F^{\prime}\left(x_{1}, x_{2}, x_{3}\right)$ which is exterior to all of the given spherical regions must be such that $V$ satisfies the following inequalities: $\sigma_{j} S_{j}(V)>0$ for $j=1,2,3$ and

$$
\frac{E(V)}{\prod_{j=1}^{3} S_{j}(V)}=-\left[\frac{N_{1} N_{2} \tau_{12}}{S_{1}(V) S_{2}(V)}\right]-\left[\frac{N_{1} N_{3} \tau_{13}}{S_{1}(V) S_{3}(V)}\right]-\left[\frac{N_{3} N_{2} \tau_{23}}{S_{2}(V) S_{3}(V)}\right] \leqq 0 .
$$

If the second inequality is multiplied through by $\prod_{j=1}^{3} \sigma_{j} S_{j}(V)>0$, it becomes

$$
\sigma_{1} \sigma_{2} \sigma_{3} E(V)=-\sigma_{1} \sigma_{2} \sigma_{3}\left[-n_{2} n_{3} \tau_{23} S_{1}(V)-n_{1} n_{3} \tau_{13} S_{2}(V)+n_{1} n_{2} \tau_{12} S_{3}(V)\right] .
$$

It is clear that the boundary surface $E(v)=0$ is again a sphere.

Since the conditions for Lemma III are satisfied (6.1) holds.

$$
0=\sum_{k=1}^{\delta_{3}} \frac{M_{k}}{V-v_{k}}=\sum_{k=1}^{\delta_{1}} \frac{m_{k}}{V-v_{k}}+\sum_{k=1+\delta_{1}}^{\delta_{2}} \frac{m_{k}}{V-v_{k}}-\sum_{k=1+\delta_{2}}^{\delta_{3}} \frac{m_{k}}{V-v_{k}} .
$$

Nagy [6] proved the following Laguerre-type theorem: "If the $Q_{j}$, $j=1,2, \cdots, p$, are points in the spherical region $\sigma S(v) \leqq 0, \sigma= \pm 1, S(v)$ $\equiv(v-c)^{2}-r^{2}, r>0$, and $P: V$ is exterior to $\sigma S(v) \leqq 0$, it follows that

$$
\sum_{k=1}^{p} \frac{m_{k}}{V-v_{k}}=\sum_{k=1}^{p} \frac{m_{k}}{V-\gamma}=\frac{n}{V-\gamma},
$$

where $m_{k}>0$ and real, $n=\sum_{k=1}^{p} m_{k}$, and $\gamma$ is the position vector of a point in $\sigma S(v) \leqq 0$.

In short, if all the zeros $Q_{j}$ of $F$ lie in a spherical region $\delta$, they may be coalesced at at least one point $Q$ in that region without altering the value of the logarithmic derivative of $F$. This theorem indicates that there exists a point $Q_{j}: \psi_{j}$ in each of the spherical regions $\sigma_{j} S_{j}(v) \leqq 0, j=1,2,3$, such that (6.2) holds.

$$
\frac{n_{j}}{V-\psi_{j}}=\sum_{k=\delta_{j-1}+1}^{\delta_{j}} \frac{m_{k}}{V-v_{k}}, \quad n_{j}=\sum_{k=\delta_{j-1}+1}^{\delta_{j}} m_{k}, \quad \delta_{0}=0, \quad j=1,2,3 .
$$

(6.1) becomes $n_{1} /\left(V-\psi_{1}\right)+n_{2} /\left(V-\psi_{2}\right)=n_{3} /\left(V-\psi_{3}\right)$ where $\psi_{j}$ is such that $\sigma_{j} S_{j}\left(\psi_{j}\right) \leqq 0$. Since $n_{3}=n_{1}+n_{2}$, this expression may be rewritten in the form 


$$
\frac{1}{V-\psi_{1}}+\left(\frac{n_{2}}{n_{1}}\right) \frac{1}{V-\psi_{2}}=\left(1+\frac{n_{2}}{n_{1}}\right) \frac{1}{V-\psi_{3}}
$$

which is equivalent to (6.3)

$$
\begin{gathered}
0=\left\{\left(V-\psi_{1}\right)^{2}+\lambda\left(V-\psi_{2}\right)^{2}-(1+\lambda)\left(V-\psi_{3}\right)^{2}\right\} V+\left(V-\psi_{3}\right)^{2} \psi_{1} \\
+\lambda\left(V-\psi_{3}\right)^{2} \psi_{2}-\left\{\left(V-\psi_{1}\right)^{2}+\lambda\left(V-\psi_{2}\right)^{2}\right\} \psi_{3} . \\
\lambda=\frac{n_{2}\left(V-\psi_{1}\right)^{2}}{n_{1}\left(V-\psi_{2}\right)^{2}}>0 .
\end{gathered}
$$

(6.3) is a relation of the type $\alpha V+\sum_{i=1}^{3} \beta_{i} \psi_{i}=0$ where $\alpha+\sum_{i=1}^{3} \beta_{i}=0$. This leads one to the conclusion that the points $P: V$, and $Q_{i}: \psi_{i}, i=1,2,3$ lie in the same plane $\Pi$. If complex numbers are introduced into $\Pi$ and $z, z_{1}, z_{2}$, and $z_{3}$ represent $P, Q_{1}, Q_{2}$, and $Q_{3}$ respectively, (6.3) becomes $-n_{2} / n_{1}$ $=\left(z-z_{2}\right)\left(z_{3}-z_{1}\right) /\left(z-z_{1}\right)\left(z_{3}-z_{2}\right)$. That is, the cross ratio formed by the points $P$ and $Q_{i}, i=1,2,3$ is a constant and the region of space bounded by $E(v)=0$ is the spherical region described by a point $P: V$ which moves so as to form a constant cross ratio, $-n_{2} / n_{1}$, with the points $Q_{j}: \psi_{j}$ as the $Q_{j}$ describes the spherical region $\sigma_{j} S_{j}(v) \leqq 0$ for $j=1,2,3$.

The final two cross ratio type theorems are generalizations of those developed by Walsh [7] for polynomials in a single complex variable.

\section{BiBLIOGRAPHY}

1. M. Bôcher, A problem in statics and its relation to certain algebraic invariants, Proc. Amer. Acad. Arts Sci. vol. 40 (1904) pp. 469-484.

2. G. Darboux, Sur une classe remarquable de courbes et de surfaces algébriques et sur la theorie des imaginaires, Paris, 1873, pp. 107-334.

3. M. Marden, On the zeros of linear partial fractions, Trans. Amer. Math. Soc. vol. 32 (1930) pp. 81-109.

4. - On the zeros of the derivative of a rational function, Bull. Amer. Math. Soc. vol. 42 (1936) pp. 400-405.

5. - The geometry of the zeros of a polynomial in a complex variable, New York, 1949.

6. G. v. Sz. Nagy, Über die Lage der Nullstellen eines Abstandspolynoms und seiner Derivierten, Bull. Amer. Math. Soc. vol. 55 (1949) pp. 329-342.

7. J. L. Walsh, On the location of the roots of the derivative of a polynomial, C.R. Congress International des Mathematiciens, Strasbourg, 1920, pp. 339-342 and C.R. Acad. Sci. Paris vol. 172 (1921) pp. 662-664.

8. - On the location of the roots of the Jacobian of two binary forms, and of the derivative of a rational function, Trans. Amer. Math. Soc. vol. 22 (1921) pp. 101-116 and vol. 24 (1922) pp. 31-69.

9. - The location of the critical points of analytic and harmonic functions, New York, 1950, pp. 109-120.

UNIVERSITY OF WisCoNSIN, Madison, Wisconsin

Iowa State Teachers College, Cedar Falls, Iowa 